\title{
Non-equilibrium effects in VECSELs
}

\author{
J. Hader \\ I. Kilen \\ S. W. Koch \\ J. V. Moloney
}




\title{
Non-equilibrium effects in VECSELs
}

\author{
J. Hader ${ }^{a}$, I. Kilen ${ }^{b}$, S. W. Koch ${ }^{a, c}$, and J. V. Moloney ${ }^{a, b, d}$ \\ ${ }^{a}$ College of Optical Sciences, The University of Arizona, \\ 1630 East University Boulevard, Tucson, Arizona 85721, USA; \\ ${ }^{b}$ Program in Applied Mathematics, University of Arizona, Tucson, Arizona 85721, USA; \\ ${ }^{c}$ Department of Physics and Material Sciences Center, Philipps-Universität Marburg, \\ Renthof 5, 35032 Marburg, Germany; \\ ${ }^{d}$ Department of Mathematics, University of Arizona, \\ 617 N. Santa Rita, Tucson, AZ 85721, USA
}

\begin{abstract}
A systematic study of microscopic many-body dynamics is used to analyze a strategy for how to generate ultrashort mode locked pulses in the vertical external-cavity surface-emitting lasers with a saturable absorber mirror. The field propagation is simulated using Maxwell's equations and is coupled to the polarization from the quantum wells using the semiconductor Bloch equations. Simulations on the level of second Born-Markov are used to fit coefficients for microscopic higher order correlation effects such as dephasing of the polarization, carrier-carrier scattering and carrier relaxation. We numerically examine recent published experimental results on mode locked pulses, as well as the self phase modulation in the gain chip and SESAM.
\end{abstract}

Keywords: VECSELs, semiconductor laser, mode-locking, simulation, semiconductor Bloch equations, RPG, SESAM, GaAs

\section{INTRODUCTION}

The vertical external-cavity surface-emitting laser (VECSEL) has proved to be versatile and has seen much research. ${ }^{1-15}$ Applications have been diverse, ranging from stable 107 fs mode locked pulsed operation to continuous-wave $(\mathrm{CW})$ operation with powers up to $106 \mathrm{~W} .^{6,7,14}$ In VECSEL cavities mode locking has been achieved using semiconductor external saturable absorber mirrors (SESAMs), graphene saturable absorbers, carbon nanotube saturable absorbers, integrated quantum well (QW) and quantum dot saturable absorbers. ${ }^{1,2,4,8-10,10-12}$ Currently, the shortest achived mode locked pulses are $107 \mathrm{fs}$ pulses ${ }^{13}$ that were compressed down to $96 \mathrm{fs},{ }^{15}$ and a train of $60 \mathrm{fs}$ pulses inside a picosecond envelope. ${ }^{3}$

A numerical model for mode locking VECSELs should be both, predictive and quantitative. Rate equation approximations first adiabatically eliminate the microscopic QW polarizations under assumptions of quasistationary carrier distributions. ${ }^{16}$ This reduces the complex QW many body dynamics into about a dozen fit parameters that have to be experimentally measured. However, the microscopic dynamics becomes increasingly important as the experimental pulses approach the ultrafast regime. This drives the need for an understanding of how the microscopic QW polarizations and non-equilibrium carrier dynamics limits mode locking operations, and whether they can potentially be used to achive even shorter mode locked pulses.

In this paper we use numerical simulations of Maxwell-semiconductor Bloch equations ${ }^{17}$ in order to examine the pulse propagation dynamics and light interaction with the QWs using a microscopic many-body theory. Simulations on the level of second Born-Markov are used to determine coefficients for the higher order correlation effects such as the polarization dephasing, carrier-carrier scattering, and carrier relaxation. The model is shown to be able to reproduce various mode locking situations such as single and multiple-pulse operation or the occurrence of interacting pulse molecules. It is used to examine microscopic limitations to achievable pulse lengths and to develop strategies for shorter pulse operation.

Further author information:

J. H.: Electronic mail: jhader@acms.arizona.edu

Vertical External Cavity Surface Emitting Lasers (VECSELs) VII, edited by Michael Jetter, Proc. of SPIE

Vol. 10087, $1008706 \cdot$ C 2017 SPIE · CCC code: 0277-786X/17/\$18 · doi: 10.1117/12.2253249 


\section{THEORETICAL BACKGROUND}

The numerical simulations of the VECSEL cavity utilizes Maxwell's equations for light propagation where the light-matter interaction, from the optically active QWs, is coupled through the polarization. For a field propagating perpendicular to the QWs it is possible to reduce Maxwell's equations into a $1 \mathrm{D}$ wave equation. Assuming the light field $E(z, t)$ propagates along the z-axis and interacts with the QWs through the macroscopic polarization $P(z, t)$ the dynamics can be determined by solving

$$
\left[\frac{\partial^{2}}{\partial z^{2}}-\frac{n(z)^{2}}{c_{0}^{2}} \frac{\partial^{2}}{\partial t^{2}}\right] E(z, t)=\mu_{0} \frac{\partial^{2}}{\partial t^{2}} P(z, t),
$$

where: $c_{0}$ is the speed of light in vacuum, $n$ is the background refractive index of the medium, and $\mu_{0}$ is the vacuum permeability. The simulation domain consists of a sequence of refractive indices that represents the different material layers. The standard boundary conditions for Maxwell's equations connects the solution in adjacent layers. Different material layers cause the propagating field to undergo reflection, transmission and phase changes depending on the sequence of refractive indices. When $E(z, t)$ interacts with the QWs it can get amplified or absorbed depending on the amount of carriers in the QWs.

The microscopic many body dynamics in the QWs are modeled by the multiband Semiconductor Bloch equations (SBE). ${ }^{17}$ These describe the time dynamics of the carrier occupation numbers, $n_{\lambda(\nu), \mathrm{k}}^{\mathrm{e}(\mathrm{h})}$, and the microscopic polarizations $p_{\lambda, \nu, \mathbf{k}}$. The SBE couple to the electric field through the macroscopic polarization $P(z, t)=\sum_{\mathbf{k}} p_{\lambda, \nu, \mathbf{k}}$.

$$
\begin{aligned}
\frac{\partial}{\partial t} p_{\lambda, \nu, \mathbf{k}} & =-\frac{\mathrm{i}}{\hbar} \sum_{\lambda_{1}, \nu_{1}}\left(e_{\lambda, \lambda_{1}, \mathbf{k}}^{\mathrm{e}} \delta_{\nu, \nu_{1}}+e_{\nu, \nu_{1}, \mathbf{k}}^{\mathrm{h}} \delta_{\lambda, \lambda_{1}}\right) p_{\lambda_{1}, \nu_{1}, \mathbf{k}}-\mathrm{i}\left(n_{\lambda, \mathbf{k}}^{\mathrm{e}}+n_{\nu, \mathbf{k}}^{\mathrm{h}}-1\right) \Omega_{\lambda, \nu, \mathbf{k}}+\Gamma_{\lambda, \nu, \mathrm{deph}}+\Lambda_{\lambda, \nu, \text { spont }}^{p}, \\
\frac{\partial}{\partial t} n_{\lambda(\nu), \mathbf{k}}^{\mathrm{e}(\mathrm{h})} & =-2 \operatorname{Im}\left(\Omega_{\lambda, \nu, \mathbf{k}}\left(p_{\lambda, \nu, \mathbf{k}}\right)^{*}\right)+\Gamma_{\lambda(\nu), \mathrm{scatt}}^{\mathrm{e}(\mathrm{h})}+\Gamma_{\lambda(\nu), \mathrm{fill}}^{\mathrm{e}(\mathrm{h})}+\Lambda_{\lambda, \nu, \mathrm{spont}}^{n} .
\end{aligned}
$$

Where $\mathbf{k}$ is the momentum of the electrons (holes) in the conduction (valanece) bands indicated by $\lambda(\nu)$. The Hartree-Fock renormalized single particle energies, $e_{\lambda, \lambda_{1}, \mathbf{k}}^{\mathrm{e}}$ and $e_{\nu, \nu_{1}, \mathbf{k}}^{\mathrm{h}}$, and the effective Rabi frequency, $\Omega_{\lambda, \nu, \mathbf{k}}$, are given by:

$$
\begin{aligned}
& e_{\lambda, \lambda_{1}, \mathbf{k}}^{\mathrm{e}}=\epsilon_{\lambda, \mathbf{k}}^{\mathrm{e}} \delta_{\lambda, \lambda_{1}}-\sum_{\lambda_{2}, \mathbf{q}} V_{|\mathbf{k}-\mathbf{q}|}^{\lambda, \lambda_{2}, \lambda_{1}, \lambda_{2}} n_{\lambda_{2}, \mathbf{q}}^{\mathrm{e}} \\
& e_{\nu, \nu_{1}, \mathbf{k}}^{\mathrm{h}}=\epsilon_{\nu, \mathbf{k}}^{\mathrm{h}} \delta_{\nu, \nu_{1}}-\sum_{\nu_{2}, \mathbf{q}} V_{|\mathbf{k}-\mathbf{q}|}^{\nu, \nu_{2}, \nu_{1}, \nu_{2}} n_{\nu_{2}, \mathbf{q}}^{\mathrm{h}}
\end{aligned}
$$

and

$$
\Omega_{\lambda, \nu, \mathbf{k}}=\omega_{\mathrm{R}}+\frac{1}{\hbar} \sum_{\lambda_{1}, \nu_{1}, \mathbf{q} \neq \mathbf{k}} V_{|\mathbf{k}-\mathbf{q}|}^{\lambda, \nu_{1}, \nu, \lambda_{1}} p_{\lambda_{1}, \nu_{1}, \mathbf{q}}
$$

Here, $e_{\lambda, \lambda_{1}, \mathbf{k}}^{\mathrm{e}}$ and $\epsilon_{\nu, \mathbf{k}}^{\mathrm{h}}$ are the single particle energies, $\omega_{\mathrm{R}}=d_{\mathbf{k}}^{\lambda, \nu} E(z, t) / \hbar$ is the Rabi frequency, $d_{\mathbf{k}}^{\lambda, \nu}$ are the dipole matrix elements, and $V_{|\mathbf{k}-\mathbf{q}|}^{\lambda, \nu_{1}, \nu, \lambda_{1}}$ is the Coulomb potential. The higher order correlation contributions that result from full second Born-Markov calculations are: the Coulomb screening, the dephasing of the polarization $\left(\Gamma_{\lambda, \nu \text {,deph }}\right)$, the carrier scattering $\left(\Gamma_{\lambda(\nu), \text { scatt }}^{\mathrm{e}(\mathrm{h})}\right)$, the kinetic hole-filling $\left(\Gamma_{\lambda(\nu), \text { fill }}^{\mathrm{e}(\mathrm{h})}\right)$, and the spontaneous emissions $\left(\Lambda_{\lambda, \nu \text {,spont }}^{n}\right)$. We will postulate a strong confinement of electrons and holes in order to simplify the SBE. In a two band approximation with parabolic bands the transition energy is related to the band gap energy, $E_{\mathrm{g}}$, and the effective masses of electrons and holes, $m_{\mathrm{e}(\mathrm{h})}$, through $\hbar \omega_{\mathbf{k}}=E_{\mathrm{g}}+\frac{\hbar^{2} \mathbf{k}^{2}}{2 m_{\mathrm{e}}}+\frac{\hbar^{2} \mathbf{k}^{2}}{2 m_{\mathrm{h}}}$.

Higher order effects are generally too time consuming to include directly into a mode locking simulation. However in the VECSEL cavity under consideration it is appropriate to assume high carrier densities in the QWs. In this situation an effective rate approximation of the correlation contributions gives good agreement 
with the full dynamics. ${ }^{18}$ Numerical simulations of the carrier scattering and polarization dephasing during a single pass of a representative mode locked pulse through a QW are used in order to determine these effective timescales. ${ }^{19}$

Under the effective rate approximation one finds three timescales, $\tau_{\text {scatt }}, \tau_{\text {deph }}$ and $\tau_{\text {fill }}$ that respectively represent the pump injection, polarization dephasing and kinetic hole filling. The models are as follows: the pump injection which leads to a relaxation toward a background Fermi distribution $f_{\lambda(\nu), \mathbf{k}}^{\mathrm{e}(\mathrm{h})}$ at the lattice tem-

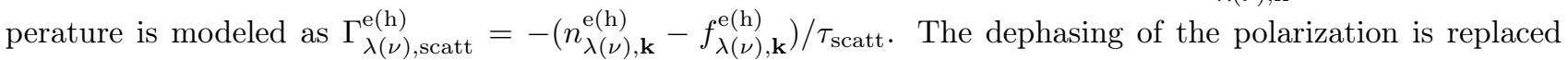
by $\Gamma_{\lambda, \nu, \text { deph }}=-\left(1 / \tau_{\text {deph }}\right) p_{\lambda, \nu, \mathbf{k}}$. The kinetic hole filling is modeled using $\Gamma_{\lambda(\nu), \text { fill }}^{\mathrm{e}(\mathrm{h})}=-\left(n_{\lambda(\nu), \mathbf{k}}^{\mathrm{e}(\mathrm{h})}-F_{\lambda(\nu), \mathbf{k}}^{\mathrm{e}}\right) / \tau_{\text {fill }}$. Here, $F_{\lambda(\nu), \mathbf{k}}^{\mathrm{e}(\mathrm{h})}$ is a quasi-Fermi distribution at the current temperature and density. In order to model spontaneous emissions we use $\Lambda_{\lambda, \nu, \text { spont }}^{n}=-\Lambda_{\mathbf{k}}^{\text {spont }} n_{\lambda, \mathbf{k}}^{e} n_{\nu, \mathbf{k}}^{h}$ and $\Lambda_{\lambda, \nu, \text { spont }}^{p}=\beta \Lambda_{\mathbf{k}}^{\text {spont }} n_{\lambda, \mathbf{k}}^{e} n_{\nu, \mathbf{k}}^{h},{ }^{20}$ with the constant $\Lambda_{\mathbf{k}}^{\text {spont }}=\frac{n^{3}}{\pi^{2} \epsilon_{0} \hbar^{4} c_{0}^{3}}\left|d_{\mathbf{k}}^{\lambda, \nu}\right|^{2}\left(E_{g}+\frac{\hbar^{2} \mathbf{k}^{2}}{2 \mathrm{~m}_{r}}\right)^{3}$.

When mode locking a VECSEL cavity the inversion, $n_{\lambda, \mathbf{k}}^{\mathrm{e}}+n_{\nu, \mathbf{k}}^{\mathrm{h}}-1$, determines what type of mode locked pulses emerges. ${ }^{21}$ The three cases are low, medium or high levels of inversion in the QWs. When the inversion is low, a single stable mode locked pulse appears, but it will not be strong enough to extract all the carriers in the QWs. When the inversion is at a medium level the pulse becomes strong enough to bleach the inversion i.e. reduce the inversion to zero at some momentum value. The pulse spectrum located at the bleached values of the QW inversion will not experience amplification. The pulse will then reshape its spectrum in order to better utilize the available inversion around the bleached areas. Finally, when the inversion is high, there are too many carriers in the QWs for a single pulse to extract. In this situation multiple pulses will form in the cavity.

Using such single pass simulations, the fully coupled microscopic Semiconductor-Maxell-Bloch model has been shown to yield very good agreement with experimental. ${ }^{22,23}$ Here, the response of the QW and SESAMs and GSAMs to pulses of various strength, widths and spectral positions has been studied and compared to time-resolved photo-luminescence measurements and experimentally measured saturation fluences.

\subsection{Simulation domain and modeling}

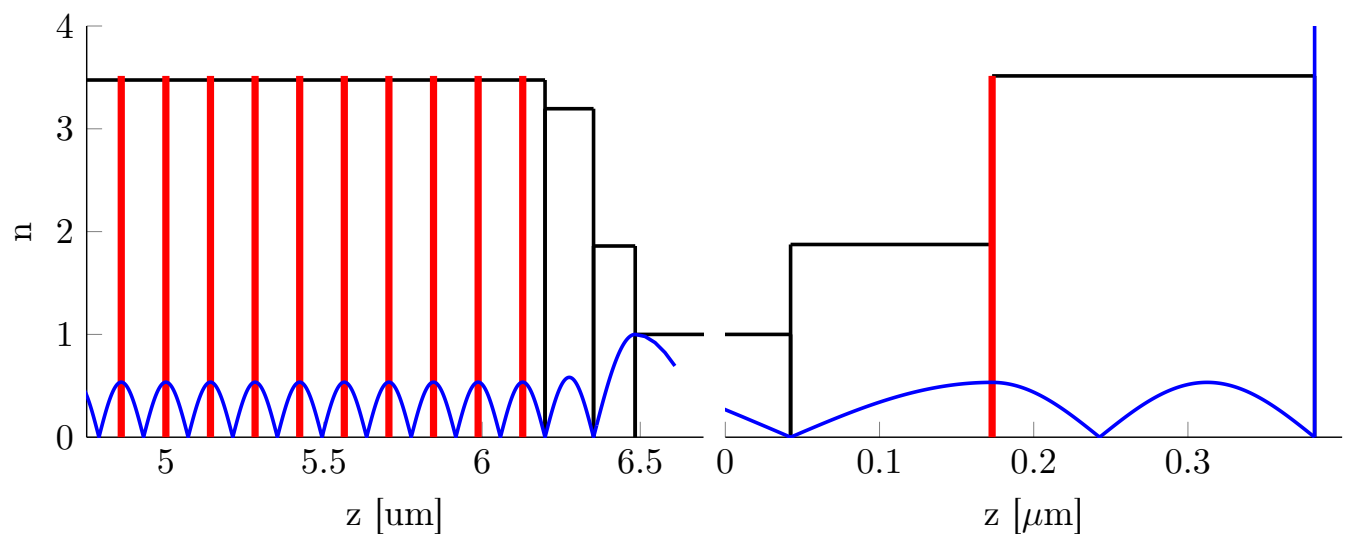

Figure 1: A cross section of a linear VECSEL cavity, and a representation of the simulation domain with the material refractive index on the vertical axis. The red lines represent the QWs and the blue curve a standing wave at the wavelength $980 \mathrm{~nm}$. Shown on the left is the QW region of the gain chip with a cap layer, and an AR coating. The DBR is not shown. On the right is the single QW SESAM on top of an output coupling mirror.

The modeled domain is a linear VECSEL cavity that includes a gain chip on top of an AlGaAs distributed Bragg reflector (DBR), an air gap and a SESAM with an output coupler. A cross section of the cavity along the direction of the propagating pulse is shown in Fig. 1. Here the different material layers are represented with the changing refractive index. Shown on the left is the QW region of the gain chip, and on the right the SESAM with an output coupler. In this example ten $8 \mathrm{~nm}$ InGaAs QWs in the gain chip are arranged in a resonant 
periodic gain (RPG) structure and the SESAM is modeled with a single $8 \mathrm{~nm}$ QW. The system is designed for operation at $980 \mathrm{~nm}$. The intensity focus on the SESAM is ten times higher than on the gain chip. This gain structure is similar to the ones that have been used to generate high power $\mathrm{CW}$ output in the past. ${ }^{6,7}$ In order to generate a short pulsed solution a single layer anti reflection (AR) coating is applied to both the gain chip and the SESAM.

In the cavity the gain chip provides optical amplification and the SESAM absorption over a wide range of wavelengths. In our model this is controlled by the initial carrier density, which is initialized at $1.9 \cdot 10^{16} \mathrm{~m}^{-2}$ in the gain chip and $5.0 \cdot 10^{14} \mathrm{~m}^{-2}$ in the SESAM. The carriers are initialized as quasi-Fermi distributions with the given density and a temperature of $300 \mathrm{~K}$. After initialization, the integration of the coupled equations (2) and (1) evolve the system from noise until it reaches a mode locked pulse. The background quasi-Fermi distribution of the pump model is kept at the initial carrier density and temperature. The cavity round trip time is set at 21 ps, the gain chip QW carrier relaxation is $30 \mathrm{ps}$, and the SESAM QW carrier relaxation time is 2 ps.

\section{RESULTS}

\subsection{Transient pulse formation}

In a VECSEL cavity there should be more spectral amplification from the inverted QWs in the gain chip than there is loss from the rest of the cavity. The spectral properties of the gain chip and the SESAM can be probed using a low energy $\operatorname{sech}^{2}$ pulse with a wide spectrum. A numerical simulation is set up such that a single low energy pulse is passed through the chip. Then the amplification or absorption is found by taking the ratio of the pulse spectrum before and after passing through the chip. This will display any spectral change in the pulse from the passive structure, as well as the inverted QWs. On the left in Fig. 2 we can see the spectral amplification and absorption from the gain chip and the SESAM at the beginning of the mode locking simulation. There is a small region near $1.265 \mathrm{eV}$ that has net gain i.e. more spectral amplification than absorption.

The mode locking simulations of a VECSEL cavity is initialized with spontaneous emissions emanating from the inverted QWs in the gain chip. These QWs will provide optical gain for the cavity light field while it will experience absorption when interacting with the SESAM QWs. When there is more amplification in the gain chip QWs than there is absorption in the SESAM there will be some positive net gain in the cavity. In this situation there is some part of the noise spectrum that will be amplified every round trip in the cavity. Eventually this noise will become strong enough to enable stimulated emission, which will continue until a single mode locked pulse appears from the noise.
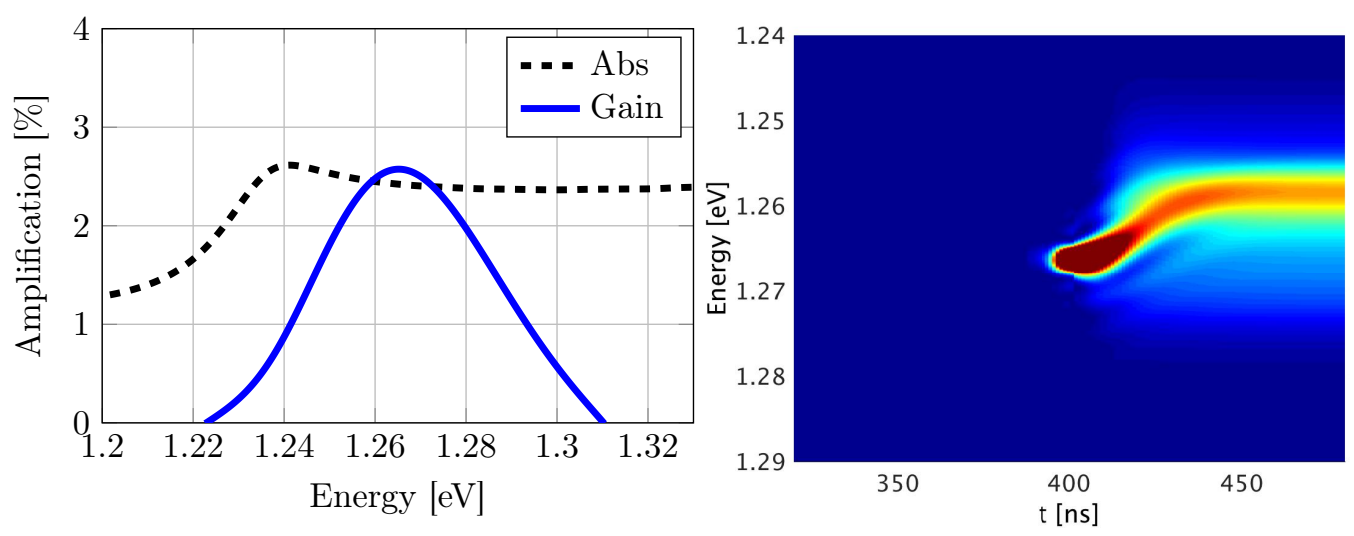

Figure 2: On the left one can see the spectral amplification and absorption of the gain chip and SESAM. On the right is s time trace of the output spectrum during the transient phase where the cavity noise develops into a mode locked pulse.

On the right in Fig. 2 one can see the spectrum of the output taken around the time that the mode locked pulse appears. In the transient phase before $400 \mathrm{~ns}$ the intensity of the stimulated emission grows because of the net gain in the cavity. This growth stops once the field intensity is strong enough to bleach out the carriers 
in the inverted QWs. At this point it is advantageous for the light field to reshape its spectrum into a wider spectral region in order to utilize all possible spectral amplification in the cavity. As a result of this, a mode locked pulse forms in the cavity. A similar temporal and spectral behavior as shown in Fig. 2 has also been observed experimentally by Turnbull et al. ${ }^{24}$

\subsection{Self phase modulation}
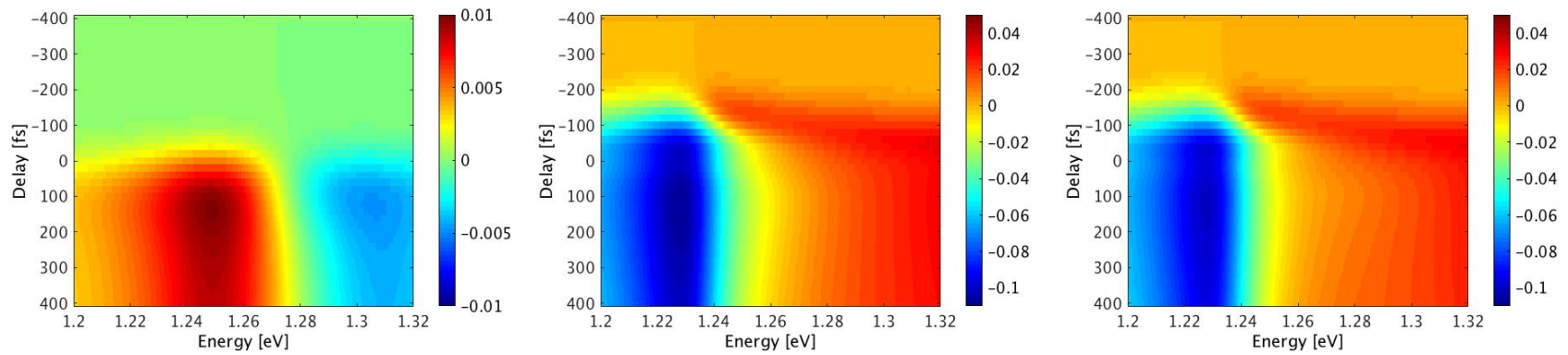

Figure 3: The refractive index change, $\Delta \mathrm{n}$, in the inverted RPG gain structure (left), absorbing SESAM (center), and sum of the refractive indices in the gain and SESAM (right) during interaction with a $220 \mathrm{fs}^{\mathrm{sech}}{ }^{2}$ pulse as measured with the Kramers-Kronig transformation. The delay is relative to when the peak of the pulse has entered the QWs. Note, the color scales are relative to each figure.

The interaction of the cavity pulse with the inverted QWs results in changes to the pulse amplitude and phase, as well as changes to QW carriers. A high-energy pulse can distort a quasi-equilibrium Fermi distribution considerably, leading to a change in the frequency dependent refractive index. In order to measure the index change, $\Delta \mathrm{n}$, one can use the Kramers-Kronig transformation which relates $\Delta \mathrm{n}$ to the change in spectral amplification. ${ }^{17}$ It is possible to measure the transient spectral amplification while the pulse interacts with the given gain chip using the probe procedure as described above. First a $220 \mathrm{fs}$ full width half maximum (FWHM) $\mathrm{sech}^{2}$ pulse centered at $1.265 \mathrm{eV}$, with similar energy to the mode locked pulse, is used to distort the QW carriers, then the spectral amplification is probed at select times around the pulse peak. This is repeated with the same pulse for the SESAM.

The refractive index change during pulse interaction in the gain chip, SESAM and the sum of the two is shown in Fig.3. The index change is most noticeable in the SESAM, where there is a low carrier density compared to the inverted QWs in the gain chip. The QW carriers in the SESAM are quickly bleached once the pulse has entered, resulting in a region where $\Delta \mathrm{n}$ is close to zero. In the combined picture we see the total refractive index change in the cavity as the pulse passes through both devices. A pulse located close to $1.265 \mathrm{eV}$ will bleach the absorber before the peak of the pulse has entered and thus the SESAM is generally called a slow saturable absorber. ${ }^{25}$ In this case the front of the pulse will experience a region of higher refractive index, or slightly lower speed of light, than the tail of the pulse. This results in the front being compressed slightly more than the rest of the pulse.

\subsection{Pulse family}

Through systematic numerical simulations we have found that the final mode locked pulse can be one of four different types ${ }^{18,22}$ : a stable and an unstable single pulse, an unstable pulse molecule or multiple pulses. For a single stable pulse, there is only one pulse propagating in the cavity and the output has constant amplitude and FWHM. (Fig 4a,c) However, in an unstable pulse the amplitude and FWHM can oscillate with a period which is much longer than the cavity round trip time. Another possible pulse shape is the pulse molecule i.e. where a longer pulse envelope contains multiple sub peaks. This shape comes from interference between two spectral peaks. (Fig 4b,d) This pulse is usually not stable because of oscillations in the FWHM and amplitude. Finally, multiple pulses will emerge in the cavity if the gain chip QWs have more inverted carriers than a single pulse is able to extract in a round trip. 

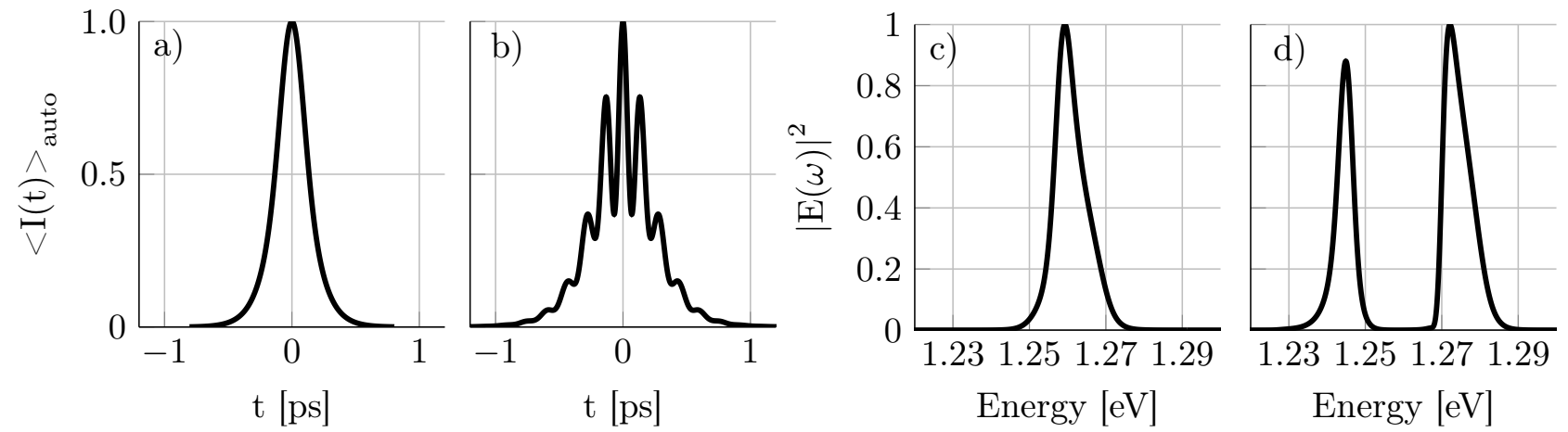

Figure 4: A sample of the final mode locked pulses that are found during simulations. The intensity autocorrelation trace of a stable single pulse and an unstable pulse molecule (ab), and their corresponding spectrums (cd).

\section{SUMMARY}

Maxwell's equations are coupled to the semiconductor Bloch equations in order to utilize a fully microscopic model for a numerical simulation of a VECSEL cavity. The transient behavior of the mode locked pulse is examined right at onset of mode locking. In the beginning, the cavity is filled with spontaneous emissions which is then amplified until the light field is strong enough to distort the carrier distributions. When the distortion is significant enough, the QW inversion is bleached. Then the spectrum of the light field expands in order to better utilize available gain. This spectral expansion also leads to the development of the mode locked pulse.

The self phase modulation caused by the changing absorption and gain spectrums is examined. In this cavity the largest contribution comes from the SESAM where the carrier density is much lower than in the inverted QWs of the gain chip.

\section{ACKNOWLEDGEMENTS}

This material is based upon work supported by the Air Force Office of Scientific Research under award number FA9550-14-1-0062.

\section{REFERENCES}

[1] Garnache, A., Hoogland, S., Tropper, A. C., Sagnes, I., Saint-Girons, G., and Roberts, J. S., "Sub-500-fs soliton-like pulse in a passively mode-locked broadband surface-emitting laser with $100 \mathrm{~mW}$ average power," Appl. Phys. Lett. 80(21) (2002).

[2] Keller, U. and Tropper, A. C., "Passively modelocked surface-emitting semiconductor lasers," Phys. Rep. 429, 67-120 (2006).

[3] Quarterman, A. H., Wilcox, K. G., Apostolopoulos, V., Mihoubi, Z., Elsmere, S. P., Farrer, I., Ritchie, D. A., and Tropper, A., "A passively mode-locked external-cavity semiconductor laser emitting 60-fs pulses," Nat. Photonics 3, 729-731 (2009).

[4] Hoffmann, M., Sieber, O. D., Wittwer, V. J., Krestnikov, I. L., Livshits, D. A., Barbarin, Y., Südmeyer, T., and Keller, U., "Femtosecond high-power quantum dot vertical external cavity surface emitting laser," Opt. Express 19, 8108-8116 (2011).

[5] Tropper, A., Quarterman, A. H., and Wilcox, K. G., [Ultrafast Vertical-External-Cavity Surface-Emitting Semiconductor Lasers], vol. 86 of Semiconductors and Semimetals, Elsevier (2012).

[6] Heinen, B., Wang, T.-L., Sparenberg, M., Weber, A., Kunert, B., Hader, J., Koch, S. W., Moloney, J. V., Koch, M., and Stolz, W., " $106 \mathrm{~W}$ continuous-wave output power from vertical-external-cavity surfaceemitting laser," Electron. Lett. 48(9), 516 (2012).

[7] Wang, T.-L., Heinen, B., Hader, J., Dineen, C., Sparenberg, M., Weber, A., Kunert, B., Koch, S. W., Moloney, J. V., Koch, M., and Stolz, W., "Quantum design strategy pushes high-power vertical-externalcavity surface-emitting lasers beyond 100 W," Laser Photon. Rev. 6(5), L12-L14 (2012). 
[8] Scheller, M., Wang, T.-L., Kunert, B., Stolz, W., Koch, S. W., and Moloney, J. V., "Passively modelocked vecsel emitting $682 \mathrm{fs}$ pulses with $5.1 \mathrm{~W}$ of average output power," Electron. Lett. 48(10), 588-589 (2012).

[9] Wilcox, K. G., Tropper, A. C., Beere, H. E., Ritchie, D. A., Kunert, B., Heinen, B., and Stolz, W., " 4.35 kW peak power femtosecond pulse mode-locked vecsel for supercontinuum generation," Opt. Express 21(2), 1599-1605 (2013).

[10] Zaugg, C., Sun, Z., Wittwer, V. J., Popa, D., Milana, S., Kulmala, T. S., Sundaram, R. S., Mangold, M., Sieber, O. D., Golling, M., Lee, Y., Ahn, J. H., Ferrari, A. C., and Keller, U., "Ultrafast and widely tuneable vertical-external-cavity surface-emitting laser, mode-locked by a graphene-integrated distributed bragg reflector," Opt. Express 21, 31548-31559 (2013).

[11] Husaini, S. and Bedford, R. A., "Antiresonant graphene saturable absorber mirror for mode-locking VECSELs." personal communication (2013).

[12] Seger, K., Meiser, N., Choi, S. Y., Jung, B. H., Yeom, D.-I., Rotermund, F., Okhotnikov, O., Laurell, F., and Pasiskevicius, V., "Carbon nanotube mode-locked optically-pumped semiconductor disk laser," Opt. Express 21, 17806-17813 (2013).

[13] Klopp, P., Griebner, U., Zorn, M., and Weyers, M., "Pulse repetition rate up to $92 \mathrm{GHz}$ or pulse duration shorter than 110 fs from a mode-locked semiconductor disk laser," Appl. Phys. Lett. 98, 071103 (2011).

[14] Laurian, A., Mart, C., Hader, J., Moloney, J. V., Kunert, B., and Stolz, W., "15 W single frequency optically pumped semiconductor laser with sub-megahertz linewidth," IEEE Photon. Technol. Lett. 26(2), 131-133 (2014).

[15] Waldburger, D., Link, S. M., Mangold, M., Alfieri, C. G. E., Gini, E., Golling, M., Tilma, B. W., and Keller, U., "High-power 100 fs semiconductor disk lasers," Optica 3(8) (2016).

[16] Sieber, O. D., Hoffmann, M., Wittwer, V. J., Mangold, M., Golling, M., Tilma, B. W., Südmeyer, T., and Keller, U., "Experimentally verified pulse formation model for high-power femtosecond VECSELs," Appl. Phys. B 113, 133-145 (2013).

[17] Haug, H. and Koch, S. W., [Quantum Theory of the Optical and Electronic Properties of Semiconductors], World Scientific, Singapore, $5^{\text {th }}$ ed. (2009).

[18] Kilen, I., Koch, S. W., Hader, J., and Moloney, J. V., "Fully microscopic modeling of mode locking in microcavity lasers," JOSAB 33(1) (2015).

[19] Hader, J., Koch, S. W., and Moloney, J. V., "Microscopic theory of gain and spontaneous emission in GaInNAs laser material," Solid-State Electron 47(3), 513-521 (2003).

[20] Bäumner, A., Koch, S. W., and Moloney, J. V., "Non-equilibrium analysis of the two-color operation in semiconductor quantum-well lasers," Phys. Status Solidi B 248(4), 843-846 (2011).

[21] Kilen, I., Hader, J., Moloney, J. V., and Koch, S. W., "Ultrafast nonequilibrium carrier dynamics in semiconductor laser mode locking," Optica 1(4), 192-197 (2014).

[22] Hader, J., Scheller, M., Laurain, A., Kilen, I., Baker, C., Moloney, J., and Koch, S., "Ultrafast nonequilibrium carrier dynamics in semiconductor laser mode-locking," Appl. Phys. B 73 (2001).

[23] Hader, J., Yang, H.-J., Scheller, M., Moloney, J., and Koch, S., "Microscopic analysis of saturable absorbers: Semiconductor saturable absorber mirrors versus graphene," Journal Appl. Phys. 119 (2016).

[24] Turnbull, A. P., Head, C. R., Shaw, E. A., Chen-Sverre, T., and Tropper, A. C., "Spectrally resolved pulse evolution in a mode-locked vertical-external-cavity surface-emitting laser from lasing onset measurements," SPIE 9349 (2015).

[25] Paschotta, R. and Keller, U., "Passive mode locking with slow saturable absorbers," Semicond. Sci. Technol. 32 (2017). 